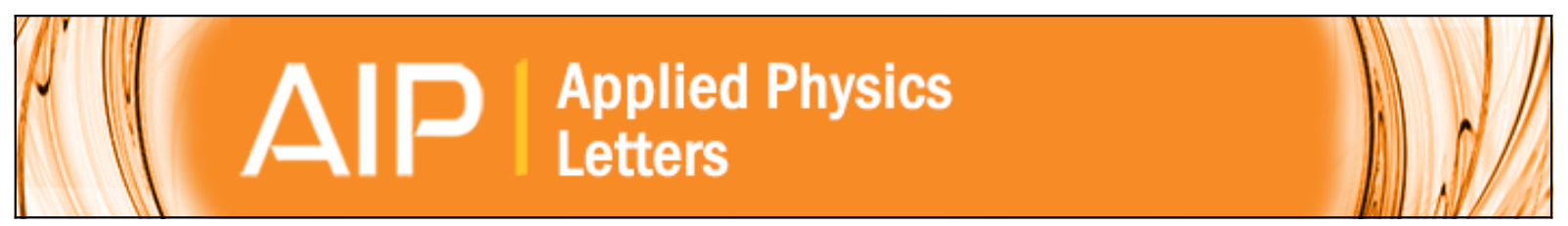

\title{
Turn on of new electronic paths in Fe-SiO2 granular thin film
}

M. A. S. Boff, R. Hinrichs, B. Canto, F. Mesquita, D. L. Baptista, G. L. F. Fraga, and L. G. Pereira

Citation: Applied Physics Letters 105, 143112 (2014); doi: 10.1063/1.4898094

View online: http://dx.doi.org/10.1063/1.4898094

View Table of Contents: http://scitation.aip.org/content/aip/journal/apl/105/14?ver=pdfcov

Published by the AIP Publishing

\section{Articles you may be interested in}

Anomalous Hall effects in Co2FeSi Heusler compound films and Co2FeSi-Al2O3 granular films

J. Appl. Phys. 111, 083919 (2012); 10.1063/1.4707846

Structure and magnetotransport properties of $\mathrm{Fe} 3 \mathrm{O} 4$ - SiO 2 composite films reactively sputtered at room temperature

J. Appl. Phys. 95, 5661 (2004); 10.1063/1.1702097

Magnetotransport and Mössbauer study of Fe $3 \mathrm{O} 4$ /y Fe $2 \mathrm{O} 3$ granular thin films

J. Appl. Phys. 89, 7693 (2001); 10.1063/1.1358351

Standing spin waves in granular Fe-SiO 2 thin films

J. Appl. Phys. 87, 5627 (2000); 10.1063/1.372471

Negative magnetoresistance of $\mathrm{Y}$ - Fe $2 \mathrm{O} 3$ observed in $\mathrm{\gamma}$ - Fe $2 \mathrm{O} 3$ /Ag granular nanocomposites

Appl. Phys. Lett. 74, 2522 (1999); 10.1063/1.123899

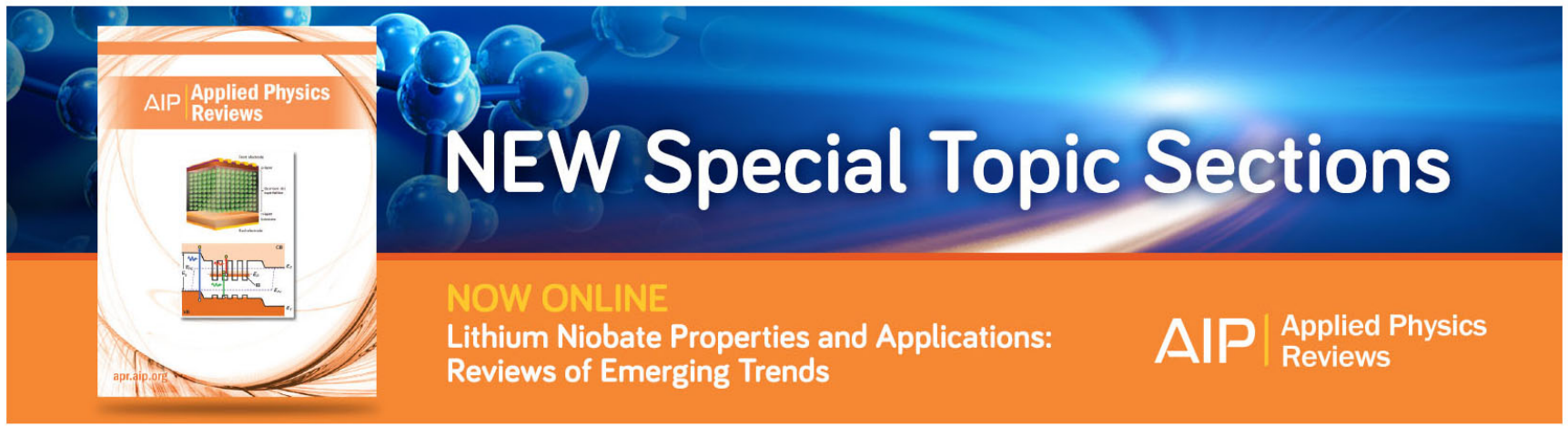




\title{
Turn on of new electronic paths in $\mathrm{Fe}-\mathrm{SiO}_{2}$ granular thin film
}

\author{
M. A. S. Boff, ${ }^{1, a)}$ R. Hinrichs, ${ }^{1,2}$ B. Canto, ${ }^{1}$ F. Mesquita, ${ }^{1}$ D. L. Baptista, ${ }^{1,3}$ G. L. F. Fraga, ${ }^{1}$ \\ and L. G. Pereira ${ }^{1}$ \\ ${ }^{1}$ Physics Institute-IF-UFRGS, C.P. 15051, 91501-970, Porto Alegre, Rio Grande do Sul, Brazil \\ ${ }^{2}$ Geosciences Institute-IG-UFRGS, C.P. 15001, 91501-970, Porto Alegre, Rio Grande do Sul, Brazil \\ ${ }^{3}$ Materials Metrology Division, INMETRO, 25250-020 Duque de Caxias, Rio de Janeiro, Brazil
}

(Received 11 September 2014; accepted 2 October 2014; published online 10 October 2014)

\begin{abstract}
The electrical properties of $\mathrm{Fe}-\mathrm{SiO}_{2}$ have been studied in the low-field regime (e $\left.\Delta \mathrm{V} \ll \mathrm{k}_{\mathrm{B}} \mathrm{T}\right)$, varying the injected current and the bias potential. Superparamagnetism and a resistance drop of $4400 \Omega$ (for a voltage variation of $15 \mathrm{~V}$ ) were observed at room temperature. This resistance drop increased at lower temperatures. The electrical properties were described with the "Mott variable range hopping" model explaining the behavior of the electrical resistance and the electronic localization length as due to the activation of new electronic paths between more distant grains. This non-ohmic resistance at room temperature can be important for properties dependent of electrical current (magnetoresistance, Hall effect, and magnetoimpedance). (C) 2014 AIP Publishing LLC.
\end{abstract}

[http://dx.doi.org/10.1063/1.4898094]

Composites that consist of metallic grains embedded in an insulating matrix are generally known as metal/insulator granular films. The structural, electrical, and magnetic properties of these thin films have been extensively investigated by many research groups,${ }^{1-3}$ and it has been shown that, in these systems, the electrical conductivity is due to electrons tunneling between grains and that magnetic and electrical behaviors change significantly when the metal volume fraction is modified. ${ }^{1}$

Metal/insulator granular thin films have two regimes of electronic transport: the low-field regime, where the electrical potential energy between grains is much lower than the thermal energy, and the high-field regime, where this potential energy is higher or equal to the thermal energy. ${ }^{1}$

Interesting effects in metal/insulator granulars were reported when the applied current was changed in the low field regime. ${ }^{9}$ The electrical resistance decreased with increasing current (or potential) for $\mathrm{Fe}-\mathrm{Al}_{2} \mathrm{O}_{3}{ }^{10}$ and $\mathrm{Co}-\mathrm{Al}_{2} \mathrm{O}_{3}{ }^{11}$ granular thin films, and the behavior was well described by Mott variable range hopping. ${ }^{4-8}$

The magnetic and electrical properties of $\mathrm{Fe}-\mathrm{SiO}_{2}$ granular thin films have been intensively studied ${ }^{12-15}$ and a non-ohmic behavior was reported $;{ }^{15}$ however, no detailed data were presented. One of the main interests in $\mathrm{Fe}_{-} \mathrm{SiO}_{2}$ granular thin film is the possibility to change several properties that depend on electrical current (magnetoresistance, ${ }^{16}$ Hall effect, and magnetoimpedance). Until now, there was not an extensive study of non-ohmic behavior in $\mathrm{Fe}-\mathrm{SiO}_{2}$ granular thin films.

In this work, we have compared the electrical resistances $(R)$ and localization length $(\xi)$ of $\mathrm{Fe}-\mathrm{SiO}_{2}$ granular film as a function of the injected current and the bias potential in the low-field regime, and explain the non-ohmic change in the electrical properties as a result of Mott variable range hopping.

The $\mathrm{Fe}-\mathrm{SiO}_{2}$ granular sample was deposited by co-sputtering at room temperature onto glass. Rutherford

\footnotetext{
${ }^{\text {a)} E l e c t r o n i c ~ a d d r e s s e s: ~ r s 014676 @ v i a-r s . n e t ~ a n d ~ m a r c o a u r e l i o s i l v e i r a b o f f ~}$ @gmail.com
}

backscattering spectrometry (RBS) showed that the metal volume fraction $(x)$ is 0.22 . Grazing incidence $\mathrm{x}$-ray diffractometry (GIXRD), with an incidence angle of $2^{\circ}$ in Seemann-Bohlin geometry of a diffractometer (Shimadzu $\mathrm{XRD6000)}$ using $\mathrm{Cu}-\mathrm{K} \alpha$ radiation showed that the iron particles were crystalline $\alpha-\mathrm{Fe}$, with a mean grain size of $8-9 \AA$, estimated from the width of the main diffraction peak using the Scherrer formula. If the distribution of grains in the sample is homogeneous, the distance between grains $(s)$, can be estimated from $x=(\pi / 6) /(1+(s / d))^{3},{ }^{1}$ where $x$ is the metal volume fraction and $d$ is the diameter of the grains. The calculated distance is $\sim 3 \AA$.

The magnetization $(M)$ was obtained using an alternating gradient field magnetometer at room temperature. The electrical resistance experiments were carried out using the four-point method with a distance of approximately $2 \mathrm{~mm}$ between the electrical contacts. Direct current was injected parallel to the sample plane. The resistance $(R)$ vs. voltage $(V)$ measurements were performed in the range from 0 to $15 \mathrm{~V}$. The resistance as a function of temperature $(T)$ was measured between $80 \mathrm{~K}$ and $300 \mathrm{~K}$. The highest potential between the contacts separated by $2 \mathrm{~mm}$ was $15 \mathrm{~V}$, placing the measurements in the low-field regime.

The magnetization curve in Fig. 1 indicates superparamagnetic behavior at room temperature, establishing the presence of ferromagnetic grains in the insulator. In superparamagnetism, there is no hysteresis, the magnetization curve is given by a Langevin function, i.e., $M=M_{0} \operatorname{coth}\left(\mu H / K_{B} T\right)-K_{B} T / \mu H$, where $\mu$ is the magnetic moment of grains, $H$ is the magnetic field, and $M_{0}$ is the saturation magnetization.

Room temperature $R$ vs. $V$ measurements are shown in Fig. 2. It can be seen that the resistance decreased with increasing voltage. The resistance variation was extremely high at room temperature, approximately $4400 \Omega$ for a voltage variation of $15 \mathrm{~V}$. The variations formerly observed in $\mathrm{Fe}-\mathrm{Al}_{2} \mathrm{O}_{3}$ granular $^{10}$ were even higher (around $7000 \Omega$ for $10 \mathrm{~V}$ at RT) than the observed in the present study. The Co$\mathrm{Al}_{2} \mathrm{O}_{3}{ }^{11}$ system showed an analogous behavior, but with lower resistance variation. 


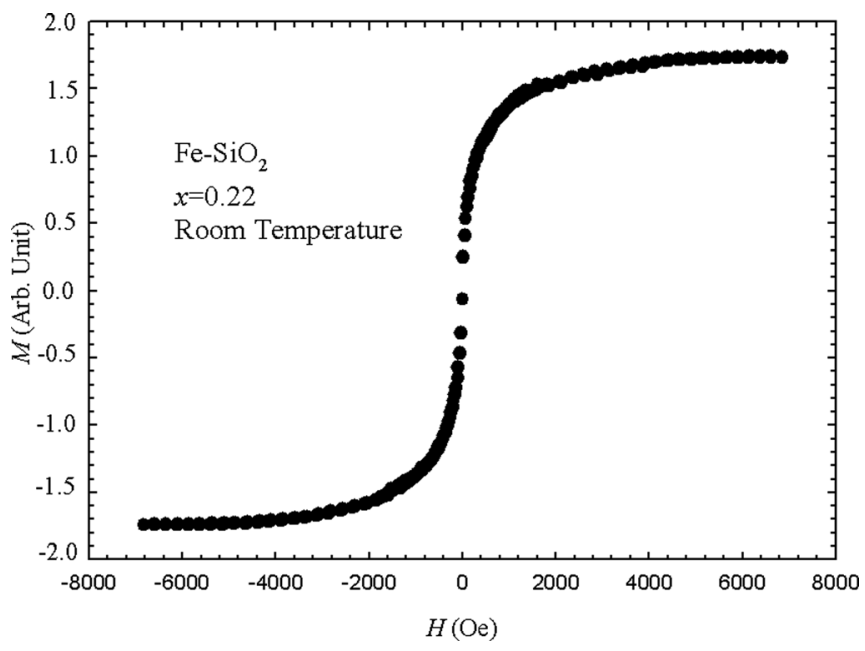

FIG. 1. $M$ as function of $H$ at room temperature of the $\mathrm{Fe}_{-} \mathrm{SiO}_{2}$ sample with $x=0.22$.

As in the other granular films, in the present case, the results are well described by Mott variable range hopping, where for a determined electron pathway, the total electrical resistance is the sum of the resistances of the individual hopping steps. In Mott's model, the resistance $R$ varies with the $1 / 4$ power of the inverse temperature (T), according to Eq. (1)

$$
R=R_{0} \exp \left(\frac{T_{0}}{T}\right)^{1 / 4}
$$

where $R_{0}$ and $T_{0}$ are appropriate constants. In Mott variable range hopping, ${ }^{5,6}$ the distance between two metal grains $\left(r_{i j}\right)$ can be correlated to the localization length $(\xi)$ as $r_{i j} \approx \xi$ $\left(T_{0} / T\right)^{1 / 4}$, while $T_{0}=\beta \Delta / N \xi^{3}$, where $\beta$ is a numerical factor, $N$ is the number of randomly distributed localized states in a band with energy width $\Delta$, centered at the Fermi level $\varepsilon_{F}$.

The $R$ vs $T$ measurements at $300 \mu \mathrm{A}$ and $500 \mu \mathrm{A}$ are shown in Fig. 3, as a $\operatorname{Ln} R$ vs. $T^{-1 / 4}$ plot that allows better visualization of the Mott $T^{-1 / 4}$ behavior. The solid lines are the fit using Eq. (1) from 100 to $150 \mathrm{~K}\left(0.31\right.$ to $\left.0.29 \mathrm{~K}^{-1 / 4}\right)$ and extrapolating for all temperatures. This fitting is very

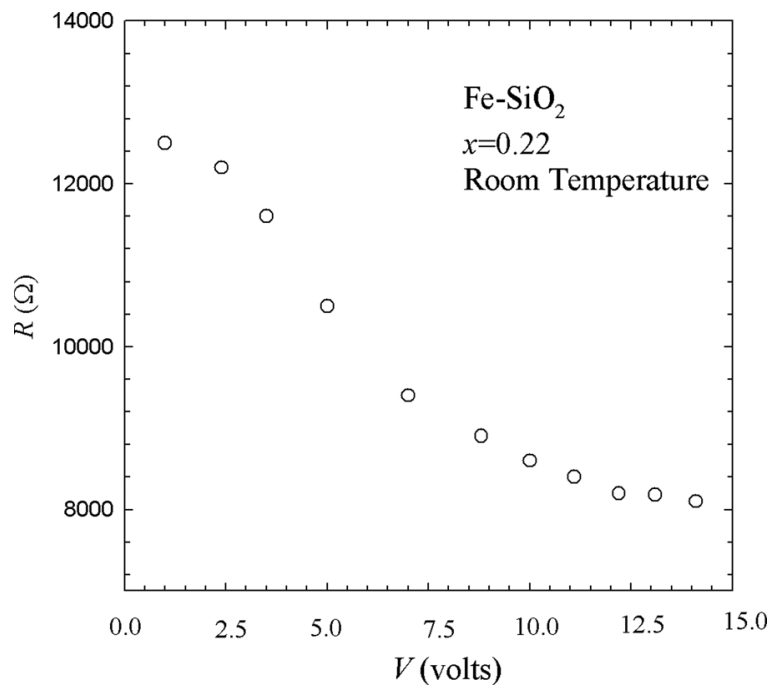

FIG. 2. Room temperature $R$ vs. $V$ curves of the $\mathrm{Fe}-\mathrm{SiO}_{2}$ sample with $x=0.22$.

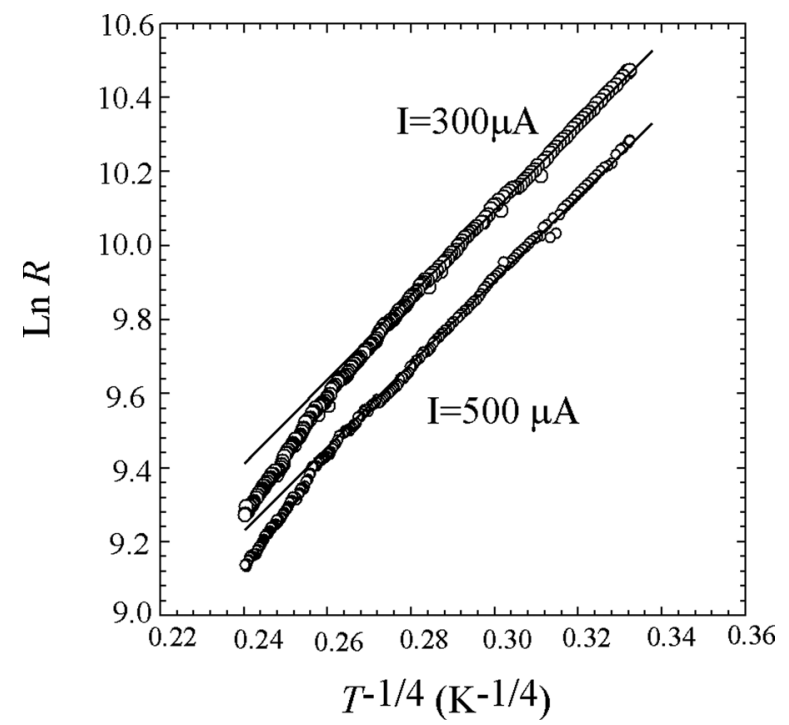

FIG. 3. Ln $R$ vs. $T^{-1 / 4}$ curve for sample $\mathrm{Fe}-\mathrm{SiO}_{2}$ with $x=0.22$. The solid lines are least squares fittings of the experimental data between $100 \mathrm{~K}$ and $150 \mathrm{~K}$ using Eq. (1).

good for temperatures below $150 \mathrm{~K}\left(0.29 \mathrm{~K}^{-1 / 4}\right)$, but for temperatures higher than $190 \mathrm{~K}\left(0.27 \mathrm{~K}^{-1 / 4}\right)$ it deviates, suggesting that a different $T_{0}$ is necessary for temperatures above $190 \mathrm{~K}$.

Because of the non-ohmic behavior of the sample, we realized $R$ vs $T$ measurements with direct currents of $100 \mu \mathrm{A}$, $200 \mu \mathrm{A}, 300 \mu \mathrm{A}$, and $500 \mu \mathrm{A}$. The fitting of the experimental data using Eq. (1) between 100 and $150 \mathrm{~K}$ is shown in Table I, indicating $T_{0}$ and the percentual variation of $T_{0}$ when compared to the $100 \mu \mathrm{A}$ value. The observed behavior was similar to that in $\mathrm{Fe}-\mathrm{Al}_{2} \mathrm{O}_{3}$ and $\mathrm{Co}-\mathrm{Al}_{2} \mathrm{O}_{3}$ granular thin films, ${ }^{10,11}$ even though the variations of $T_{0}$ were not as pronounced as in those.

The $R$ vs $T$ plot of the $\mathrm{Fe}-\mathrm{SiO}_{2}$ sample using $300 \mu \mathrm{A}$ and $500 \mu \mathrm{A}$ is displayed in Fig. 4 and shows that the resistance is systematically smaller at all temperatures when the current is higher. In our hypothesis of new parallel pathways, this corresponds to the addition of a parallel resistance, which is approximately 4-6 times bigger than the resistance between neighboring grains. The thermal behavior of this parallel resistance, however, is the same as the resistance at lower current, because the percentage of reduction is maintained throughout the temperature range.

When new paths are enabled between more distant grains, $r_{i j}$ increases and each path has higher resistance. Since the new paths, even though more resistant, are turned on in parallel, the overall resistance $R$ drops.

In Fig. 5, an idealized situation is depicted, where the grains are hexagonally packed in a plane, with regular

TABLE I. $T_{0}$ temperature for different electrical currents.

\begin{tabular}{lcc}
\hline \hline Direct current $(\mu \mathrm{A})$ & $T_{0}(\mathrm{~K})$ & $\left(T_{0}(i)-T_{0}(100 \mu \mathrm{A}) / T_{0}(100 \mu \mathrm{A}) \times 100(\%)\right.$ \\
\hline 100 & 22271 & 0 \\
200 & 18842 & -15.4 \\
300 & 17064 & -23.4 \\
500 & 15987 & -28.2 \\
\hline
\end{tabular}




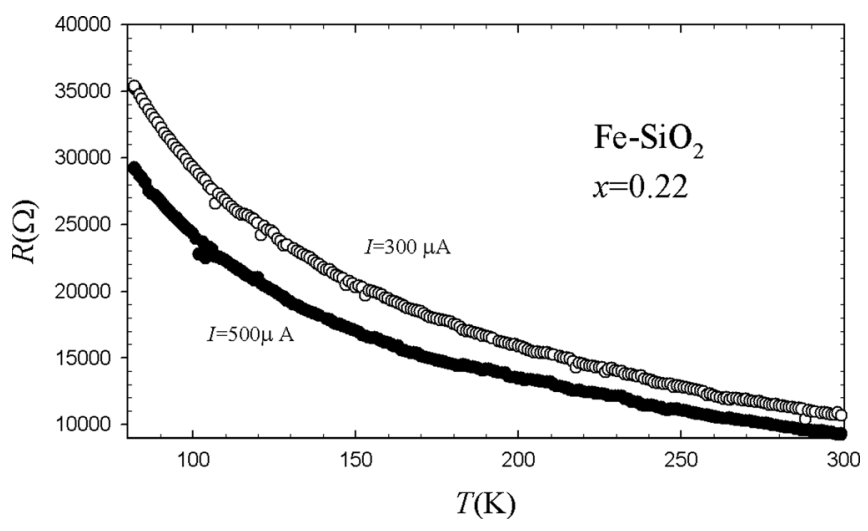

FIG. 4. $R$ vs. $T$ curves for the $\mathrm{Fe}-\mathrm{SiO}_{2}$ sample, using injected currents of $300 \mu \mathrm{A}$ and $500 \mu \mathrm{A}$. The Ln $R$ vs. $T^{-1 / 4}$ curves are presented.

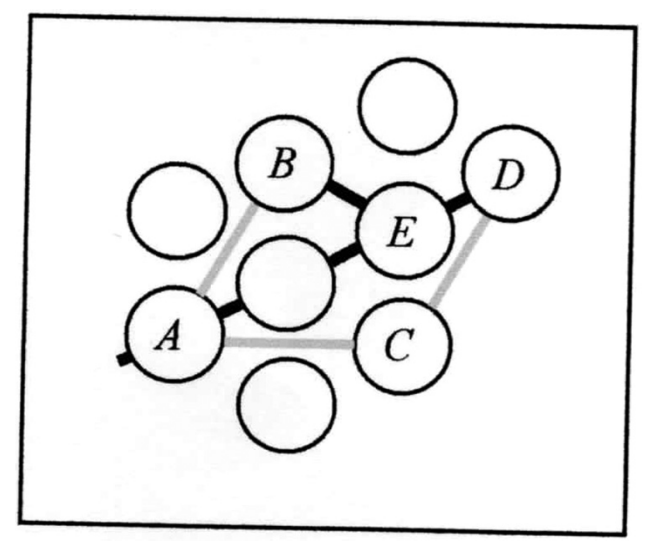

FIG. 5. Idealized view of a densely packed plane of equally distributed spheres of equal size with two different pathways: at lower currents, only paths between nearest neighbors are active (black); while at higher currents, a pathway between second nearest neighbors can be activated (grey/grey or grey/black).

distances between them (roughly proportional to the dimensions calculated for $x=0.22$ ). The black lines indicate electronic pathways between nearest neighbors (nn), while the grey lines correspond to new pathways between second nearest neighbors (snn), which are only activated at higher currents (or higher potential). In a hexagonal plane lattice with $9 \AA$ spheres separated by $3 \AA$, the distance between second nearest neighbors is $\sim 3$ times the distance between nearest neighbors. Considering a path from the grains $A$ to $D$, with the activation of a new path involving one snn and one nn (via grain $B$ ) a decrease of $19 \%$ is achieved, while the path involving two snn grains (via grain $C$ ) reduces the resistance in $27 \%$. If after approximately every $10 \mathrm{nn}$ hoppings, one parallel path involving a snn was activated, the overall reduction would be around $10 \%$. This would be plausible, if the size of the spheres was distributed around a mean, implying that the distances between snn would be a distribution as well, where the shorter distances would be favored as new parallel paths first.

This physical description of the electronic pathways explains the increase of resistance variation with current or bias potential at lower temperatures as well. If a new path is turned on in parallel at room temperature, the total resistance of the sample decreases. If the new path is turned on at a lower temperature, it will have a higher resistance and the overall resistance variation will be higher.

Our study of $\mathrm{Fe}-\mathrm{SiO}_{2}$ granular thin film showed that:

When the current and or bias potential are increased in the low-field regime, $R$ and $T_{0}$ decreased and, using the Mott variable range hopping interpretation, the decrease of $T_{0}$ was associated with an increase of the localization length;

The resistance variation as a function of the current or the bias potential was higher at lower temperatures;

The non-ohmic behavior of the metal/insulator granular thin films in the low-field regime was interpreted as being associated to new electronic paths between more distant grains, which decreased the total resistance of the sample and increased the localization length $\xi$.

${ }^{1}$ B. Abeles, Applied Solid State Science: Advances in Materials and Device Research, edited by R. Wolfe (Academic, New York, 1976), p. 1.

${ }^{2}$ K. M. Unruth and C. L. Chien, Nanomaterials: Synthesis, Preparation, and Application, edited by A. S. Edelstein and A. C. Camarata (1996), p. 347.

${ }^{3}$ C. L. Chien, Annu. Rev. Mater. Sci. 25, 129 (1995).

${ }^{4}$ P. Sheng, B. Abeles, and Y. Arie, Phys. Rev. Lett. 31, 44 (1973).

${ }^{5}$ N. F. Mott, J. Non-Cryst. Solids 1, 1 (1968).

${ }^{6}$ B. I. Shklovskii and B. Z. Spivak, in Hopping Transport in Solids, Modern Problems in Condensed Science Vol. 28, edited by M. Pollak and B. Shklovskii (Elsevier Science, Amsterdam, 1991), Chap. 9.

${ }^{7}$ K. Yakushiji, S. Mitani, F. Ernult, K. Takanashi, and H. Fujimori, Phys. Rep. 451, 1 (2007).

${ }^{8}$ S. H. Ge, S. B. Zhang, J. H. Chi, Z. G. Zhang, C. X. Li, and R. J. Gan, J. Phys. D 33, 917 (2000).

${ }^{9}$ M. A. S. Boff, S. R. Teixeira, J. E. Schmidt, and A. B. Antunes, Appl. Phys. Lett. 85, 757 (2004).

${ }^{10}$ M. A. S. Boff, F. Casarin, L. G. Pereira, and A. B. Antunes, Physica B 406, 1833 (2011).

${ }^{11}$ M. A. S. Boff, B. Canto, R. Hinrichs, L. G. Pereira, F. Mesquita, J. E. Schmidt, and G. L. Fraga, Physica B 406, 4304 (2011).

${ }^{12}$ S. Honda, T. Okada, and M. Nawate, Phys. Rev. B 56, 14566 (1997).

${ }^{13}$ L. Xi, Z. Zhang, J. Wang, C. Li, F. Li, S. Ge, T. Xu, and S. Yang, J. Phys. D 33, 621 (2000).

${ }^{14}$ W. Yang, Z. S. Jiang, W. N. Wang, and Y. W. Du, Solid State Commun. 104(8), 479 (1997).

${ }^{15}$ J. Leveneur, J. Kennedy, G. V. M. Williams, J. Metson, and A. Markwitz, Appl. Phys. Lett. 98, 053111 (2011).

${ }^{16}$ M. A. S. Boff, J. Geshev, J. E. Schmidt, W. H. Flores, A. B. Antunes, M. A. Gusmão, and S. R. Teixeira, J. Appl. Phys. 91(12), 9909 (2002). 